EPJ Web of Conferences 72, 00005 (2014)

DOI: $10.1051 /$ epjconf / 20147200005

C) Owned by the authors, published by EDP Sciences, 2014

\title{
Studies of Continuum Physics at KLOE/KLOE-2
}

\author{
Veronica De Leo ${ }^{1,2, *}$ for the KLOE/KLOE-2 Collaborations ${ }^{\dagger}$ \\ ${ }^{1}$ Università di Messina, Dipartimento di Fisica e Scienze della Terra, via F.S.D'Alcontres, 98166 Messina, \\ Italy \\ ${ }^{2}$ INFN, Sezione di Catania, via Santa Sofia 64, 95123 Catania, Italy
}

\begin{abstract}
The measurement of the ratio $\sigma\left(e^{+} e^{-} \rightarrow \pi^{+} \pi^{-} \gamma\right) / \sigma\left(e^{+} e^{-} \rightarrow \mu^{+} \mu^{-} \gamma\right)$ was performed with the KLOE detector at $D A \Phi N E$, the Frascati $\phi$-factory, using events with initial state radiation photons emitted at small angle and inclusive of the final state radiation. The analysis is based on a data set corresponding to an integrated luminosity of $240 \mathrm{pb}^{-1}$. The cross section $\sigma\left(e^{+} e^{-} \rightarrow \pi^{+} \pi^{-}\right)$, obtained from the ratio, has been used to determine the pion form factor $\left|F_{\pi}\right|^{2}$ and the two pion contribution to the muon anomaly $a_{\mu}$ for $0.592<M_{\pi \pi}<0.975 \mathrm{GeV}$. The result confirms the previous KLOE measurements and the current discrepancy between the Standard Model calculation and the experimental value of the muon anomaly. The preliminary results on the combination of the last analysis (KLOE12) with the previous published (KLOE08,KLOE10) will be also presented.
\end{abstract}

*e-mail:vdeleo@unime.it

$\dagger$ KLOE-2 collaboration: D. Babusci, I. Balwierz-Pytko, G. Bencivenni, C. Bloise, F. Bossi, P. Branchini, A. Budano, L. Caldeira Balkestảhl, G. Capon, F. Ceradini, P. Ciambrone, F. Curciarello, E. Czerwiński, E. Danè, V. De Leo, E. De Lucia, G. De Robertis, A. De Santis, P. De Simone, A. Di Cicco, A. Di Domenico, C. Di Donato, R. Di Salvo, D. Domenici, A. D’Uffizi, O. Erriquez, G. Fanizzi, A. Fantini, G. Felici, S. Fiore, P. Franzini, A. Gajos, P. Gauzzi, G. Giardina, S. Giovannella, E. Graziani, F. Happacher, L. Heijkenskjöld B. Höistad, L. Iafolla, M. Jacewicz, T. Johansson, K. Kacprzak, D. Kamiǹska, A. Kupsc, J. Lee-Franzini, B. Leverington, F. Loddo, S. Loffredo, G. Mandaglio, M. Martemianov, M. Martini, M. Mascolo, R. Messi, S. Miscetti, G. Morello, D. Moricciani, P. Moskal, F. Nguyen, A. Palladino, A. Passeri, V. Patera, I. Prado Longhi, A. Ranieri, C. F. Redmer, P. Santangelo, I. Sarra, M. Schioppa, B. Sciascia, M. Silarski, C. Taccini, L. Tortora, G. Venanzoni, W. Wis̀licki, M. Wolke, J. Zdebik.

This is an Open Access article distributed under the terms of the Creative Commons Attribution License 4.0, which permits unrestricted use, distribution, and reproduction in any medium, provided the original work is properly cited. 


\section{Introduction}

The Dirac equation predicts a muon magnetic moment, $\vec{M}=g_{\mu} \frac{e}{2 m_{\mu}} \vec{S}$, with gyromagnetic ratio $g_{\mu}=2$. Quantum loop effects lead to a small calculable deviation from $g_{\mu}=2$, parameterized by the anomalous magnetic moment

$$
a_{\mu} \equiv \frac{g_{\mu}-2}{2} .
$$

That quantity can be accurately measured and, within the Standard Model (SM) framework, precisely predicted [1]. Measurements of the muon magnetic anomaly $a_{\mu}=\left(g_{\mu}-2\right) / 2$ performed at the Brookhaven Laboratory have reached an accuracy of $0.54 \mathrm{ppm}: a_{\mu}=$ $(11659208.9 \pm 6.3) \times 10^{-10}[1,2]$. The quoted value differs from Standard Model estimates by 3.2 - 3.6 standard deviations [3-7]. A deviation in $a_{\mu}^{\text {exp }}$ from the SM expectation would signal effects of new physics, reaching mass scales of $O(\mathrm{TeV})$ with current sensitivity[8, 9]. The SM prediction for $a_{\mu}^{S M}$ is generally divided into three parts (see Fig. 1 for representative Feynman diagrams):

$$
a_{\mu}^{S M}=a_{\mu}^{Q E D}+a_{\mu}^{E W}+a_{\mu}^{H a d}
$$
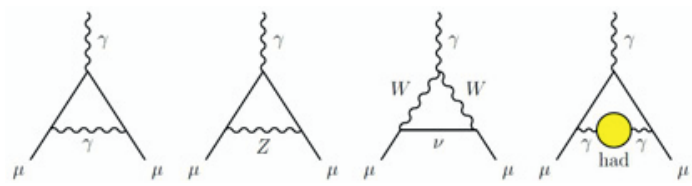

Figure 1. Representative diagrams contributing to $a_{\mu}^{S M}$. From left to right: first order QED (Schwinger term), lowest-order electro-weak, lowest order hadronic.

The QED part includes all photonic and leptonic $(\mathrm{e}, \mu, \tau)$ loops starting with the classic $\alpha / 2 \pi$ Schwinger contribution. Loop contributions involving heavy $W \pm, Z$ or Higgs particles are collectively labeled as $a_{\mu}^{E W}$. Hadronic (quark and gluon) loop contributions to $a_{\mu}^{S M}$ give rise to its main theoretical uncertainties [1]. The hadronic contribution itself is given by different contributions:

$$
a_{\mu}^{\mathrm{Had}}=a_{\mu}^{\mathrm{Had}, L O}+a_{\mu}^{\mathrm{Had}, H O}+a_{\mu}^{\mathrm{Had}, L B L}
$$

where $a_{\mu}^{H a d, L O}$ is the lowest-order contribution from hadronic vacuum polarization, $a_{\mu}^{\mathrm{Had}, \mathrm{HO}}$ is the corresponding higher-order part and the last term $a_{\mu}^{\mathrm{Had}, L B L}$ is the light-by-light (LBL) scattering part [9]. The dominant hadronic terms can be calculated with a combination of experimental cross section data, related to $e^{+} e^{-}$annihilation to hadrons and perturbative QCD. These terms are used to evaluate an energy-squared dispersion integral:

$$
a_{\mu}^{H a d}[L O]=\frac{1}{3}\left(\frac{a}{\pi}\right)^{2} \int_{m_{\pi}^{2}}^{\infty} d s \frac{K(s)}{s} R(s)
$$

where $K(s)$ is a QED kernel function [10], and where $R(s)$ denotes the ratio of the cross section for $e^{+} e^{-}$annihilation into hadrons to the pointlike muon-pair cross section at center of mass energy $\sqrt{s}$. The integration kernels occurring in the dispersion relations emphasise low photon virtualities, due to the $1 / \mathrm{s}$ slope of the cross section.

At $D A \Phi N E$ the differential cross section for $e^{+} e^{-} \rightarrow \pi^{+} \pi^{-} \gamma$ for initial state radiation (ISR) events is measured. Then, the dipion cross section $\sigma_{\pi \pi} \equiv \sigma\left(e^{+} e^{-} \rightarrow \pi^{+} \pi^{-}\right)$has been obtained from:

$$
\left.s \frac{d \sigma\left(\pi^{+} \pi^{-} \gamma\right)}{d \sigma_{\pi}}\right|_{I S R}=\sigma_{\pi \pi}\left(s_{\pi}\right) H\left(s_{\pi}, s\right),
$$

where the radiator function $H$ is computed from QED with complete NLO corrections [11$15]$ and depends on the $e^{+} e^{-}$center of mass energy squared $s . \quad \sigma_{\pi \pi}$ obtained from Eq. 5 requires accounting for final state radiation (FSR). Eq. 5 is also valid for $e^{+} e^{-} \rightarrow \mu^{+} \mu^{-} \gamma$ and $e^{+} e^{-} \rightarrow \mu^{+} \mu^{-}$with the same radiator function $H$. We can therefore determine $\sigma_{\pi \pi}$ from the ratio of the $\pi^{+} \pi^{-} \gamma$ and $\mu^{+} \mu^{-} \gamma$ differential cross sections for the same value of the dipion and dimuon invariant mass (see also Refs. [16, 17]).

The pion form factor is calculated by the following equation: 
$\left|F_{\pi}\left(s^{\prime}\right)\right|^{2}=\frac{3}{\pi} \frac{s^{\prime}}{\alpha^{2} \beta_{\pi}^{3}} \sigma_{\pi \pi(\gamma)}^{0}\left(s^{\prime}\right)\left(1+\delta_{V P}\right)\left(1-\eta_{\pi}\left(s^{\prime}\right)\right)$

where $\delta_{V P}$ is the VP correction [18], $\eta_{\pi}$ accounts for FSR radiation assuming point-like pions [19] and $\sigma_{\pi \pi}^{0}$ is the bare cross section defined as:

$$
\begin{aligned}
& \sigma^{0}\left(\pi^{+} \pi^{-}, s^{\prime}\right)= \\
& \frac{d \sigma\left(\pi^{+} \pi^{-} \gamma, I S R\right) / d s^{\prime}}{d \sigma\left(\mu^{+} \mu^{-} \gamma, I S R\right) / d s^{\prime}} \times \sigma^{0}\left(e^{+} e^{-} \rightarrow \mu^{+} \mu^{-} \gamma, s^{\prime}\right)
\end{aligned}
$$

where $s^{\prime}=s_{\pi}=s_{\mu}$.

Many radiative corrections drop out using the ratio method. In particular those ones due to the radiator function (avoiding the related systematic uncertainty of $0.5 \%$ in the $\sigma_{\pi \pi}$ measurement), to the integrated luminosity (since the data sample for the $\pi^{+} \pi^{-} \gamma$ and $\mu^{+} \mu^{-} \gamma$ events are the same) and finally to the vacuum polarization.

\section{KLOE Detector}

The KLOE detector operates at $D A \Phi N E$, the Frascati $\phi$-factory, an $e^{+} e^{-}$collider running at fixed energy, $W=\sqrt{s} \sim 1020 \mathrm{MeV}$, the $\phi$ meson mass.

It consists of a cylindrical drift chamber (DC) [20] and an electromagnetic calorimeter (EMC) [21]. The DC has a momentum resolution of $\sigma_{p_{\perp}} / p_{\perp} \sim 0.4 \%$ for tracks with polar angle $\theta>45^{\circ}$. Track points are measured in the DC with a resolution in $r-\phi$ of $\sim 0.15 \mathrm{~mm}$ and $\sim 2$ $\mathrm{mm}$ in z. The EMC has an energy resolution of $\sigma_{E} / E \sim 5.7 \% / \sqrt{E}(\mathrm{GeV})$ and an excellent time resolution of $\sigma_{t} \sim 54 \mathrm{ps} / \sqrt{E}(\mathrm{GeV}) \oplus 100 \mathrm{ps}$.

Calorimeter clusters are reconstructed grouping together energy deposits close in space and time. A superconducting coil provides an axial magnetic field of $0.52 \mathrm{~T}$ along the bisector of the colliding beam directions. The bisector is taken as the $\mathrm{z}$ axis of our coordinate system. The $\mathrm{x}$ axis is horizontal, pointing to the center of the collider rings and the $y$ axis is vertical, directed upwards. A cross section of the detector in the $\mathrm{y}, \mathrm{z}$ plane is shown in

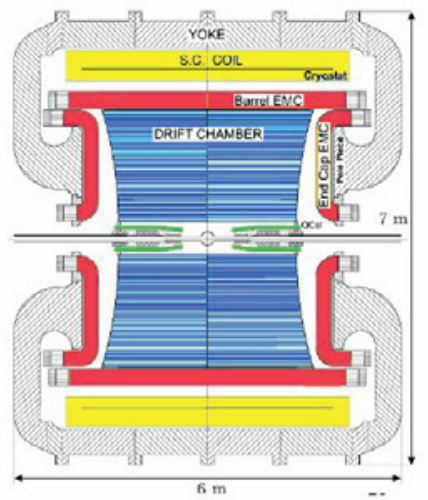

Figure 2. Schematic view of the KLOE detector. In the picture the superconducting coil (yellow), the electromagnetic calorimeter (red) and the cylindrical drift chamber (blue) are visible.

Fig.2. The $\pi^{+} \pi^{-} \gamma$ cross section diverges at small photon angle as $\left(1-\cos ^{2} \theta_{\gamma}\right)^{-2} \propto 1 / \theta_{\gamma}^{4}$ making FSR $\pi^{+} \pi^{-} \gamma$ processes and $\phi$ decays relatively unimportant. For this reason we measure $d \sigma / d M_{\pi \pi}^{2}$ at small photon angle.

\section{Measurement of the $e^{+} e^{-} \rightarrow \pi^{+} \pi^{-}$ cross section normalized to luminosity from Bhabha events}

In the 2008 and 2010 two measurements of the $\sigma\left(e^{+} e^{-} \rightarrow \pi^{+} \pi^{-} \gamma\right)$ have been performed at $D A \Phi N E$ with the KLOE detector.

The KLOE08 analysis [22] used selection cuts in which the photon is emitted within a cone of $\theta_{\gamma}<15^{\circ}$ around the beamline (narrow cones in Fig.3) and the two charged pion tracks have $50^{\circ}<\theta_{\pi}<130^{\circ}$ (wide cones in Fig. 3). In this configuration, the photon is not explicitly detected so the photon momentum is reconstructed from kinematics: $\overrightarrow{p_{\gamma}} \simeq \overrightarrow{p_{\text {miss }}}=-\left(\overrightarrow{p_{+}}+\overrightarrow{p_{-}}\right)$. These selection cuts allowed to have high statistics for ISR signal events, and a reduced contamination from the resonant process $e^{+} e^{-} \rightarrow \phi \rightarrow$ $\pi^{+} \pi^{-} \pi^{0}$.

Using Eq. 5 the pion form factor $\left|F_{\pi}\right|^{2}$ is extracted. From the bare cross section, i.e. corrected for the running of $\alpha_{e m}$ and inclusive 


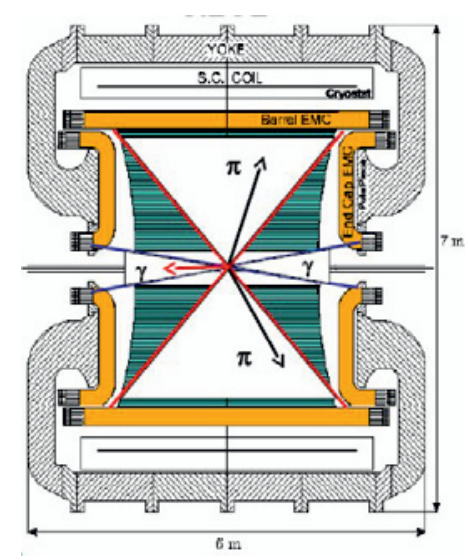

Figure 3. Schematic view of the KLOE detector with selection regions.

of FSR, the dipion contribution to the muon anomaly $\Delta^{\pi \pi} a_{\mu}$ is measured:

$$
\left.\underset{(387.2 \pm 3.3) \times 10^{-10} .}{\Delta^{\pi \pi} a_{\mu}(0.592<} M_{\pi \pi}<0.975 \mathrm{GeV}\right)=
$$

The KLOE10 analysis [23] was performed requiring events that are selected to have a photon at large polar angles between $50^{\circ}<\theta_{\gamma}<$ $130^{\circ}$ (wide cones in Fig.3), in the same angular region as the pions.

This selection cut allowed to access the two pion threshold. However, compared to the measurement with photons at small angles, this condition implied a reduction in statistics and an increase of the background from the process $\phi \rightarrow$ $\pi^{+} \pi^{-} \pi^{0}$. The following value for the dipion contribution to the muon anomaly $\Delta^{\pi \pi} a_{\mu}$ was found:

$$
\Delta^{\pi \pi} a_{\mu}(0.1-0.85) G e V^{2}=\left(478.5 \pm 2.0_{\text {stat }} \pm\right.
$$
$\left.5.0_{\text {exp }} \pm 4.5_{t h}\right) \times 10^{-10}$.

In the fig. 4 the comparison between the measurements of the pion form factor of the KLOE08 and KLOE10 analysis is reported.

As one can see, an excellent agreement is found for $\left(M_{\pi \pi}^{0}\right)^{2}>0.5 G e V^{2}$, while below the KLOE10 result is lower by few percent. We stress that the data sets have been obtained at different operating conditions of the DAФNE collider, and different selection cuts in acceptance were used. Also the analysis procedures were different since in the KLOE08 analysis the radiated photon was not detected.
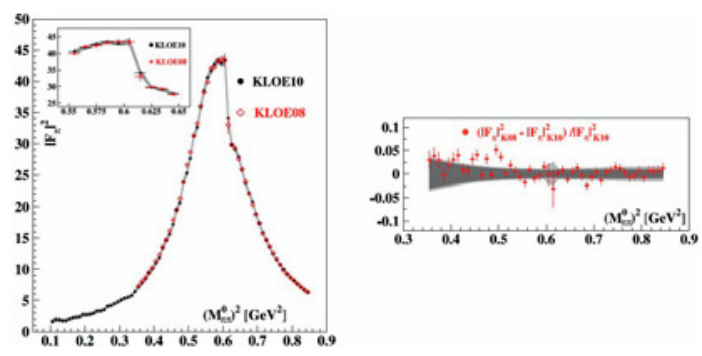

Figure 4. Comparison of KLOE10 result with the previous KLOE08 result [22]. Left: Pion form factor $\left|F_{\pi}\right|^{2}$. Right: Fractional difference between KLOE08 and KLOE10 results. The dark (light) gray band gives the statistical (total) error for the present result. Errors on KLOE08 points contain the combined statistical and systematic uncertainty.

In Fig.5, the KLOE10 result is compared with the results from the energy scan experiments CMD-2 [24, 25] and SND [26] in Novosibirsk and the result obtained from the BaBar experiment at SLAC [27], which uses the ISR method. Whenever several data points fall in one KLOE bin of $0.01 G e V^{2}$, the values are statistically averaged. In the Fig. 5, left, the comparison of $\left|F_{\pi}\right|^{2}$ obtained by the CMD-2 and SND collaborations with the KLOE10 result is shown [23].

The agreement with the SND result is rather good around the $\rho$-peak and at higher energies, while the result from the CMD- 2 collaboration is slightly higher than the new KLOE measurement, confirming the observation already reported in the previous KLOE publication [22]. Below the $\rho$-peak, all three experiments are in agreement within uncertainties. In the Fig.5, right, KLOE10 and the BaBar result for the bare cross section as a function of $M_{\pi \pi}^{0}$ is shown. The fractional difference between $\mathrm{BaBar}$ and KLOE results is shown together with the statistical and total fractional KLOE errors. The two results are in agreement within errors below 0.65 $\mathrm{GeV}$, while above the new BaBar measurement is about $2-3 \%$ higher. 

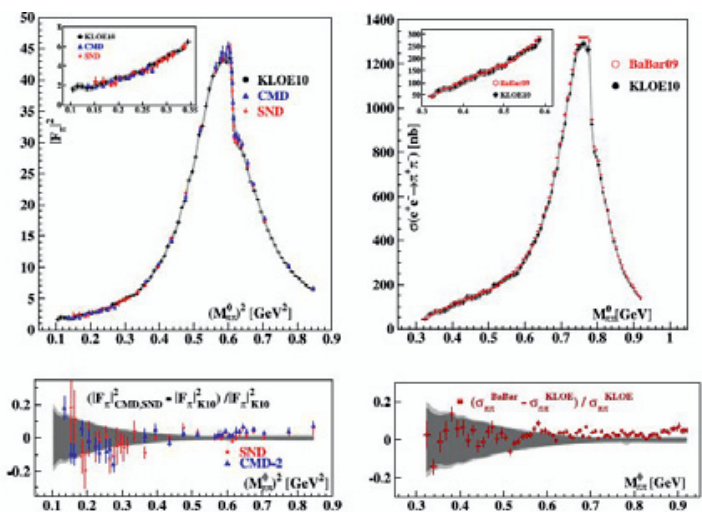

Figure 5. Top left: $\left|F_{\pi}\right|^{2}$ from CMD-2 [24, 25], SND [26] and KLOE10 result as function of $\left.M_{\pi \pi}^{0}\right)^{2}$. Bottom left: Fractional difference between CMD-2 or SND and KLOE. Top right: $\sigma_{\pi \pi}^{\text {bare }}$ from BaBar [27] and the new KLOE result as function of $M_{\pi \pi}^{0}$. Bottom right: Fractional difference between $\mathrm{BaBar}$ and KLOE. CMD-2, SND and BaBar data points have the total uncertainty attached. The dark (light) band in the lower plots shows statistical (total) error of the KLOE result [23].

\section{Measurement of the pion form factor from the $\pi \pi \gamma / \mu \mu \gamma$ ratio}

The data sample corresponds to an integrated luminosity of $239.2 \mathrm{pb}^{-1}$ collected in 2002 (the same used in the KLOE08 analysis).

The separation between the $\pi \pi \gamma$ and $\mu \mu \gamma$ events is obtained assuming the final state of two charged particles with equal mass $M_{T R K}$ and one photon: the $M_{T R K}<115 \mathrm{MeV}$ for the muons and $M_{T R K}>130 \mathrm{MeV}$ for the pions (see Fig.6 ). From the energy and momentum conservation laws we obtain:

$$
\begin{gathered}
\left(\sqrt{s}-\sqrt{\left|\overrightarrow{p_{+}}\right|^{2}+M_{T R K}^{2}}-\sqrt{\left|\overrightarrow{p_{-}}\right|^{2}+M_{T R K}^{2}}\right)^{2}- \\
+\left(\overrightarrow{p_{+}}+\overrightarrow{p_{-}}\right)^{2}=0
\end{gathered}
$$

where $\overrightarrow{p_{ \pm}}$is the measured momentum of the positive (negative) particle.

This selection leads to $8.9 \times 10^{5} \mu \mu \gamma$ events and about $34.9 \times 10^{5}$ for $\pi \pi \gamma$ events. The selection procedure has been compared to other tech-

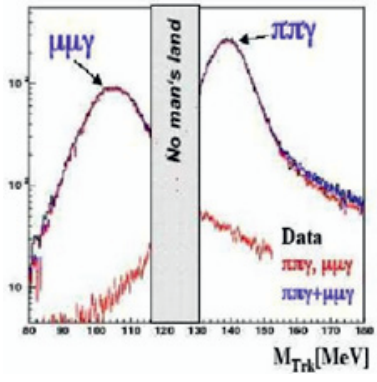

Figure 6. Separation of pion and muon events by cuts in $M_{T R K}$.

niques, such as a kinematic fit or tighter cuts on the quality of the charged tracks, all bringing to consistent results.

Trigger, particle identification and tracking efficiencies have been checked from data control samples.

The differential $\mu \mu \gamma$ cross section is obtained from the observed event count $N_{o b s}$ and background estimate $N_{b k g}$, as:

$$
\frac{d \sigma_{\mu \mu \gamma}}{d s_{\mu}}=\frac{N_{o b s}-N_{b k g}}{\Delta s_{\mu}} \frac{1}{\epsilon\left(s_{\mu}\right) L}
$$

where $L$ is the integrated luminosity from Ref. [28] and $\epsilon\left(s_{\mu}\right)$ the selection efficiency. Fig. 7 , top, shows the measured $\mu \mu \gamma$ cross section compared with the QED calculations to NLO, using the MC code Phokhara [14].

Fig.7, bottom, shows the ratio between the two differential cross sections. The green band shows the systematic uncertainty, experimental and theoretical, of the measured cross section. The average ratio, using only statistical errors, is $0.9981 \pm 0.0015$, in agreement with the quoted systematic uncertainties[29].

The bare cross section $\sigma_{\pi \pi(\gamma)}^{0}$ (inclusive of FSR, with VP effects removed) is obtained from the bin-by-bin ratio of the Ref.[22] $\pi \pi \gamma$ and the $\mu \mu \gamma$ differential cross sections described above. This cross section is used in the dispersion integral to compute $\Delta^{\pi \pi} a_{\mu}$. The pion form factor $\left|F_{\pi}\right|^{2}$ is then calculated using Eq. (6).

The dispersion integral for $\Delta^{\pi \pi} a_{\mu}$ is computed as 


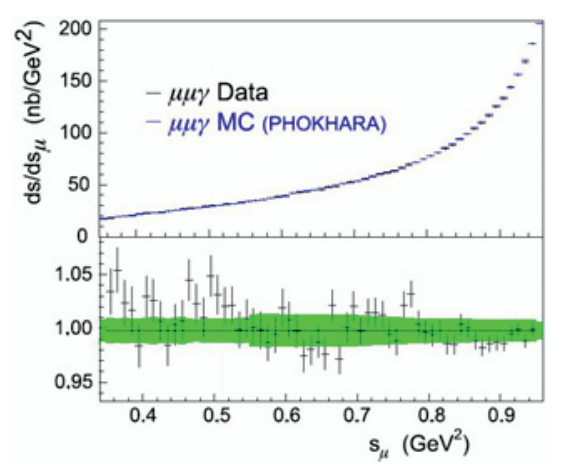

Figure 7. Top. Comparison of data and MC results for $d \sigma_{\mu \mu \gamma} / d s_{\mu}$. Bottom. Ratio of the two spectra. The green band shows the systematic error[29].

the sum of the values for $\sigma_{\pi \pi(\gamma)}^{0}$ times the kernel $\mathrm{K}(\mathrm{s})$, times $\Delta s=0.01 \mathrm{GeV}^{2}$ :

$$
\Delta^{\pi \pi} a_{\mu}=\frac{1}{4 \pi^{3}} \int_{s_{\min }}^{s_{\max }} d s \sigma_{\pi \pi(\gamma)}^{0}(s) K(s)
$$

where the kernel is given in Ref. [30]. Eq. 10 gives $\Delta^{\pi \pi} a_{\mu}=\left(385.1 \pm 1.1_{\text {stat }} \pm 2.6_{\exp } \pm 0.8_{\text {th }}\right) \times$ $10^{-10}$ in the interval $0.35<M_{\pi \pi}^{2}<0.95 \mathrm{GeV}^{2}$. For each bin contributing to the integral, statistical errors are combined in quadrature and systematic errors are added linearly.

In the table 1 the comparison between the last $\Delta^{\pi \pi} a_{\mu}$ KLOE measurement and the previous KLOE08 and KLOE10 measurements is reported.

Table 1. Comparison of $\Delta^{\pi \pi} a_{\mu}$ between the KLOE12 and the previous KLOE measurements.

\begin{tabular}{ll}
\hline Measurement & $\Delta a_{\mu}^{\pi \pi}\left(0.35-0.95 \mathrm{GeV}^{2}\right) \times 10^{10}$ \\
\hline KLOE12 & $385.1 \pm 1.1_{\text {stat }} \pm 2.7_{\text {sys }+ \text { theo }}$ \\
KLOE08 & $387.2 \pm 0.5_{\text {stat }} \pm 3.3_{\text {syst theo }}$ \\
\hline & $\Delta a_{\mu}^{\pi \pi}\left(0.35-0.85 \mathrm{GeV}^{2}\right) \times 10^{10}$ \\
\hline KLOE12 & $377.4 \pm 1.1_{\text {stat }} \pm 2.7_{\text {sys }+ \text { theo }}$ \\
KLOE10 & $376.6 \pm 0.9_{\text {stat }} \pm 3.3_{\text {sys }+ \text { theo }}$
\end{tabular}

The Fig.8 shows the good agreement between the KLOE12 and KLOE08 measurements, in particular in the $\rho$ region. An improvement of the systematic error has been

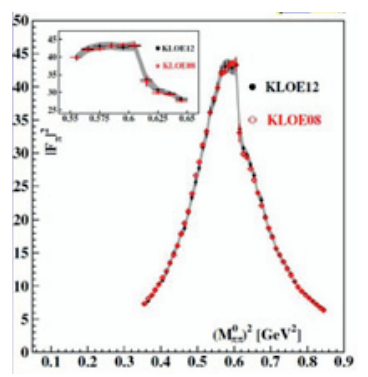

Figure 8. The pion form factor obtained in the KLOE12 (points) and KLOE08 [22] (crosses) measurements.
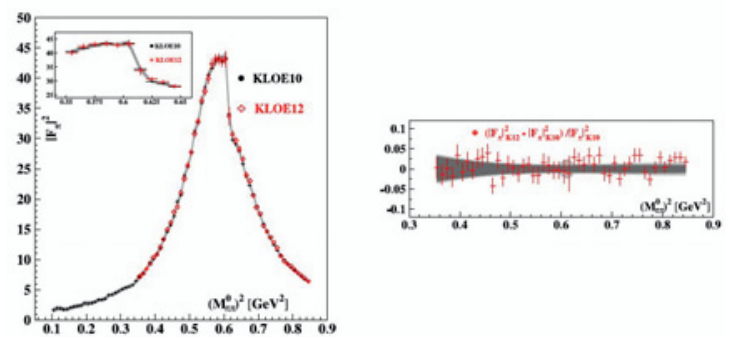

Figure 9. Left: the pion form factor obtained in this work, KLOE12 (crosses) and from the measurement with the photon at large angle, KLOE10 [23] (points). Right: fractional difference between the two $\left|F_{\pi}\right|^{2}$ measurements. The dark grey band is the statistical error from [23], the light grey band is the combined statistical and systematic uncertainty. In both figures, errors on crosses include combined statistical and systematic uncertainties.

reached in the KLOE12 measurement. A sizeable improvement of the theoretical error is achieved due to the ratio method. Fig. 9 (left) shows the comparison between the KLOE12 $\left|F_{\pi}\right|^{2}$ measurement and the previous KLOE10 [23] measurement, requiring the ISR photon to be reconstructed at large angle, inside the EMC barrel. Fig. 9 (right) shows the fractional difference between the two measurements. They are obtained from independent data sets with different running conditions ( $\mathrm{W}=M_{\phi}$ here, $\mathrm{W}=1 \mathrm{GeV}$ in Ref. [23]), and also with a different selection, that in turn imply 
independent systematic uncertainties. The two measurements are in very good agreement.

The preliminary combination of the last three KLOE results (KLOE08, KLOE10, KLOE12) is reported. It is obtained using the Best Linear Unbiased Estimate (BLUE) method [31, 32]. In the Fig. (left) the pion form factor measurements for the three KLOE analysis and the fractional difference (right) are shown. The following $a_{\pi \pi}^{\mu}$ values are found:

$$
\begin{aligned}
& a_{\pi \pi}^{\mu}\left(0.1-0.95 G e V^{2}\right)=(487.8 \pm 5.7) \cdot 10^{-10} \\
& a_{\pi \pi}^{\mu}\left(0.1-0.85 G e V^{2}\right)=(378.1 \pm 2.8) \cdot 10^{-10}
\end{aligned}
$$

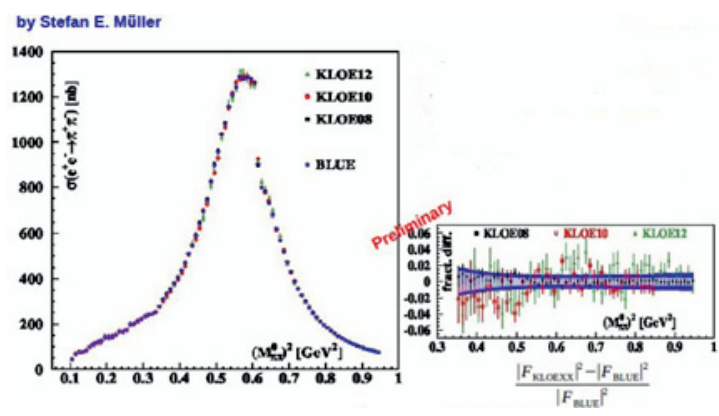

Figure 10. Preliminary combination of the last three KLOE results (KLOE08, KLOE10, KLOE12) on the pion form factor measurements (left) and the fractional difference (right) using the Best Linear Unbiased Estimate $(B L U E)$ method $[31,32]$.

\section{Conclusion}

During the last 10 years KLOE has performed a series of precision measurements with ISR which confirmed a $3 \sigma$ discrepancy between $a_{\mu}$ $\mathrm{SM}$ and the value measured at BNL. The published measurements (KLOE08, KLOE10), normalized to Bhabha events, have allowed the measurement of $a_{\mu}^{\pi \pi}$ in the region below 1 $\mathrm{GeV}$ with $\sim 1 \%$ total error. A new measurement (KLOE12) of $\left|F_{\pi}\right|^{2}$ from the $\pi \pi \gamma / \mu \mu \gamma$ ratio (based on $240 \mathrm{pb}^{-1}$ ) with $0.7 \%$ systematic error has been published [29]. This pion form factor determination is in very good agreement with previous KLOE results.

\section{References}

[1] J.Beringer et al. (Particle Data Group), PR D 86, 010001 (2012) (URL: http://pdg.lbl.gov)

[2] G.W. Bennett, et al., Muon G-2 Collaboration, Phys. Rev. D 76 (2006) 072003.

[3] J.P. Miller, E. de Rafael, B.L. Roberts, Rept. Prog. Phys. 70 (2007) 795.

[4] F. Jegerlehner, A. Nyffeler, Phys. Rept. 477 (2009) 1.

[5] M. Davier, A. Hoecker, B. Malaescu, Z. Zhang, Eur. Phys. J. C 71 (2011) 1515.

[6] M. Davier, A. Hoecker, B. Malaescu, Z. Zhang, Eur. Phys. J. C 72 (2012) 1874 (Erratum).

[7] K. Hagiwara, et al., J. Phys. G 38 (2011) 085003.

[8] A. Czarnecki and W.J. Marciano, Phys. Rev. D 64, 013014 (2001).

[9] M. Davier and W.J. Marciano, Ann. Rev. Nucl. and Part. Sci. 54, 115 (2004).

[10] S.J. Brodsky and E. de Rafael, Phys. Rev. 168, 1620 (1968).

[11] G. Rodrigo, H. Czyż , J.H. Kühn, M. Szopa, Eur. Phys. J. C 24 (2002) 71.

[12] H. Czyż, A. Grzelinska, J.H. Kühn, G. Rodrigo, Eur. Phys. J. C 27 (2003) 563.

[13] H. Czyż, A. Grzelinska, J.H. Kühn, G. Rodrigo, Eur. Phys. J. C 33 (2004) 333.

[14] H. Czyż, A. Grzelinska, J.H. Kühn, G. Rodrigo, Eur. Phys. J. C 39 (2005) 411.

[15] S. Actis, et al., Working Group on Radiative Corrections and Monte Carlo Generators for Low Energies Collaboration, Eur. Phys. J. C 66 (2010) 585.

[16] B. Aubert, et al., BaBar Collaboration, Phys. Rev. Lett.103 (2009) 231801.

[17] J.P. Lees, et al., BaBar Collaboration, Phys. Rev. D 86 (2012) 032013.

[18] VP correction values kindly provided by Fred Jegerlehner, http://wwwcom. physik.huberlin.de/ fjeger/alphaQEDn.uu.

[19] J.S. Schwinger,Particles, Sources and Fields, vol. 3, Addison-Wesley, Redwood City, USA, (1989), p. 99. 
[20] M. Adinolfi, et al., KLOE Collaboration, Nucl. Instrum. Methods A 488 (2002) 51.

[21] M. Adinolfi, et al., KLOE Collaboration, Nucl. Instrum. Methods A 482 (2002) 364.

[22] F. Ambrosino, et al., KLOE Collaboration, Phys. Lett. B 670 (2009) 285.

[23] F. Ambrosino, et al., KLOE Collaboration, Phys. Lett. B 700 (2011) 102.

[24] R.R. Akhmetshin, et al., CMD-2 Collaboration, Phys. Lett. B 648 (2007) 28.

[25] R.R. Akhmetshin, et al., CMD-2 Collaboration, JETP Lett. 84 (2006) 413.

[26] M.N. Achasov, et al., SND Collaboration, J. Exp. Theor. Phys. 103 (2006) 380.

[27] B. Aubert, et al., BaBar Collaboration, Phys. Rev. Lett. 103 (2009) 231801.

[28] F. Ambrosino, et al., KLOE Collaboration, Eur. Phys. J. C 47 (2006) 589.

[29] D.Babusci et al.,KLOE and KLOE-2 Collaborations, Physics Letters B 720 (2013) 336.

[30] B.E. Lautrup, E. de Rafael, Nuovo Cim. A 1 (1971) 238.

[31] A. Valassi NIM A 500 (2003) 391.

[32] G. D’Agostini NIM A 346 (1994) 306. 